\title{
BMJ Open Money-oriented risk-takers or deliberate decision-makers: a cross-sectional survey study of participants in controlled human infection trials
}

\author{
Marie-Astrid Hoogerwerf (D) , ${ }^{1}$ Martine de Vries, ${ }^{2}$ Meta Roestenberg ${ }^{1,3}$
}

To cite: Hoogerwerf M-A, de Vries M, Roestenberg M. Money-oriented risk-takers or deliberate decision-makers: a cross-sectional survey study of participants in controlled human infection trials. BMJ Open 2020;10:e033796. doi:10.1136/ bmjopen-2019-033796

- Prepublication history and additional material for this paper are available online. To view these files, please visit the journal online (http://dx.doi org/10.1136/bmjopen-2019033796).

Received 23 August 2019 Revised 20 April 2020 Accepted 11 June 2020

\section{Check for updates}

C Author(s) (or their employer(s)) 2020. Re-use permitted under CC BY-NC. No commercial re-use. See rights and permissions. Published by BMJ.

${ }^{1}$ Parasitology, Leiden University Medical Center, Leiden, ZuidHolland, The Netherlands

${ }^{2}$ Medical Ethics and Health Law, Leiden University Medical Center, Leiden, Zuid-Holland, The Netherlands

${ }^{3}$ Infectious Diseases, Leiden University Medical Center, Leiden, Zuid-Holland, The Netherlands

Correspondence to Dr Meta Roestenberg; m.roestenberg@lumc.nl

\section{ABSTRACT}

Objective To quantitatively investigate the motivations, decision-making and experience of participants in controlled human infection (CHI) studies.

Design Cross-sectional descriptive survey study.

Setting Previous participants of $\mathrm{CHI}$ studies at the Leiden Controlled Human Infection Center, control group of students from Leiden University.

Participants 61 previous participants and 156 controls. Measurements Ranking of motivational and decisional factors, risk propensity score and multiple-choice questions on experience of trial participation and ethical aspects of $\mathrm{CHI}$ studies.

Results Motivating factors for participants were contributing to science $(81 \%)$, contributing to research that may benefit developing countries (72\%) and the financial compensation $(63 \%)$. For $51 \%$ of participants, a reason other than financial compensation was the most important motivational factor. Participants considered trust in the study team (70\%), time investment $(63 \%)$, severity of symptoms (54\%), chance of developing symptoms $(54 \%)$ and whether it is an easy way to make money (54\%) in their decision to participate. Most CHI participants (84\%) were proud of their participation, would advise others to participate (89\%) and would participate in a similar trial again (85\%). CHI participants had a higher risk propensity score than students (estimated difference $0.9, p<0.001$ ). Conclusion Although financial compensation is important, the motivations for participants in a $\mathrm{CHI}$ study are diverse and participants make a balanced appraisal of risks and burden before participating.

\section{INTRODUCTION}

Controlled human infection (CHI) trials are increasingly used in the development of novel vaccines and drugs against a variety of pathogens. ${ }^{1}$ In these trials, volunteers are purposely infected with a pathogen to test the efficacy of new vaccines or medicines and to study host-pathogen interaction. ${ }^{2}$ CHI trials have boosted vaccine development against, for example, malaria ${ }^{3}$ and cholera, ${ }^{4}$ and generated valuable information on host-pathogen interactions in many other diseases. ${ }^{2}$ Currently, over 23000 volunteers
Strengths and limitations of this study

- First quantitative study on motivations and experiences of participants in controlled human infection (CHI) studies.

- Included multiple $\mathrm{CHI}$ models with a relatively large group of participants, increasing generalisability.

- Answers may have been biassed by recall or social desirability.

- Control group had high percentage of missing answers on questionnaires, although all questions were answered by at least $85 \%$ of controls.

- Control group consisted of students, which is a more homogenous population than the participants, of which roughly $2 / 3$ were students. This difference may hamper comparison.

have participated in these studies, ${ }^{1}$ with exponentially increasing numbers over the past decades. Like phase I drug trials also including healthy volunteers, CHI studies lack individual benefit to the volunteer, requiring a thorough review of the balance of risk and burden to the participant versus the social and scientific benefits. Literature on the ethical debate of CHI trials is growing, with particular emphasis on informed consent, undue influence by financial compensation and the right to withdraw. ${ }^{5-7}$

Like the debate concerning phase I drug trials, ${ }^{8}$ there is suspicion that volunteers are only driven by money ${ }^{910}$ and as a result do not adequately weigh the risk and burden of participation, ${ }^{11}$ the 'money-orientated risk-taker'. Participants in phase I trials score higher on questionnaires examining sensation-seeking behaviours compared with age-matched and sex-matched controls, adding to the notion that these volunteers are more prone to take, possibly ill-considered, risks in their lives. ${ }^{12} 13$ However, recent research shows that phase I participants consider other arguments besides the financial compensation, such 
as curiosity, contributing to medical research, helping future patients and the risks involved. ${ }^{14}{ }^{15}$ In response to a recent publication ${ }^{16}$ public discussion, particularly on social media, has also focussed on voluntariness of participation since studies often include medical students as participants who were presumed to have felt pressure to participate, next to the ongoing discussion about acceptability of risks and burdens. Qualitative data on motivation of participants was recently collected in two studies with volunteers in controlled human malaria infection (CHMI) trials in the USA and Kenya. These showed that participants had other motivations next to the financial incentive. ${ }^{17} 18$ However, these studies only included small groups of participants (16 and 36, respectively) in a malaria trial, and quantitative data on motivations and experiences is lacking. Given the ongoing debate on the ethics of CHI trials, a more quantitative assessment of the experiences and motivation of participants in a broader group of volunteers is needed to gain better insight into the profile of the CHI volunteer, their motivations and experiences.

In order to investigate whether participants in CHI trials are different from the general population, it is valuable to compare the participants to a control group. This also enables a longitudinal comparison of motivations and thought processes of potential participants with those who have actually participated, providing a better insight into how volunteers come to their decision. An additional benefit of a control group from the general population is that there will be a proportion unwilling to participate. These controls provide a comparator in decisional factors and can give information on the acceptance of ethical aspects of CHIs by those unwilling to take part.

The Leiden Controlled Human Infection Center has conducted multiple CHI trials in malaria, schistosomiasis and hookworm. This unique setup offers an ideal opportunity to fill the above-mentioned knowledge gaps. We therefore conducted a survey study in former participants of these trials, using students from the local university as a control group. The aim of this study is to quantitatively investigate the motivation, decision-making process and risk propensity of participants in CHI trials compared with a control group. Furthermore, this study explores participants' views on ethical questions in CHI trials.

\section{METHODS}

This cross-sectional descriptive survey was conducted among participants of CHI trials performed at the Leiden Center for Controlled Human Infections and students of the Leiden University in October 2018.

\section{Participants}

Participants of previously conducted CHI trials with malaria, hookworm or schistosomiasis were invited to participate in an anonymous survey. Inclusion criteria were having undergone $\mathrm{CHI}$ and previous consent to be contacted again for further studies. There were no exclusion criteria. All 66 previous participants were eligible for inclusion. CHI trials were conducted between November 2016 and September 2018. Surveys were distributed and collected via e-mail through data management programme Castor EDC. ${ }^{19}$ Participants who did not respond to the e-mail were sent one reminder. CHI participants received a $10 €$ voucher as reward.

As control group, students from the local university were included. This group has been selected as the majority of participants in CHI studies at the study centre is recruited from this population. Before lectures at the medical faculty, the anonymous paper survey was distributed to all students present and collected afterwards. Surveys were also distributed during two meetings of local (non-medical) student societies, where the researchers handed students present the survey and collected them after completion. Controls did not receive compensation.

With an expected response rate of $80 \%$, we estimated that around 50 previous participants would return the survey. Based on experiences in recruiting, we estimated that one-third of students would be willing to participate in a CHI trial. So, in order to include an equal number of controls willing to participate to actual participants, we aimed to include 150 controls.

\section{Survey}

The survey was designed by the researchers, based on previously published research ${ }^{14}{ }^{15}$ and topics of ethical debate. ${ }^{5}$ Motivational and decision-making factors were chosen based on the research by Grady et $a l^{15}$ and by identification of potential motivational factors through discussion with researchers involved in screening and recruitment of trial participants. Participants were allowed to add their own factors. Motivational factors in the survey were 'curiosity', 'contributing to science', 'contributing to developing countries', 'financial compensation', 'interest in the subject' and 'personal experience'. Factors in the decision-making process were 'severity of possible symptoms', 'chance of developing symptoms', 'time investment', 'an easy way to make money', 'trust in the study team' and 'it's research about parasites'. Questions on ethical acceptability were formulated based on issues identified in literature as key concepts in CHI trials ${ }^{5-7}$ (surveys in online supplementary A).

$\mathrm{CHI}$ participants (from here referred to as PP) reflected on their own experiences, whereas the control group (CC) were asked to consider participation in a malaria trial and a trial with hookworm to reflect the different types of studies conducted. CG were provided descriptions of the trials detailing study procedures, possible adverse events, number of visits and sample collections and the financial compensation (descriptions in online supplementary A). PP and CC were asked to rate motivational factors and factors considered in their decision about participation. Each factor could be rated as not important, slightly important, considerably important or very important. Next to this ranking, $\mathrm{CC}$ and $\mathrm{PP}$ were also asked to identify the single most important factor. 
Attitudes towards risk-taking were investigated using the Risk Propensity Scale (RPS), ${ }^{20}$ a seven-item questionnaire consisting of statements on taking risks in daily life that are rated between 1 and 9 (online supplementary B). Higher scores represent a higher propensity to take risks. This questionnaire was selected as this is a concise questionnaire focussing on general risk-taking propensity in daily life.

Experiences of PP and opinions on ethical issues were examined using multiple-choice questions. Wherever relevant, CC were presented with similar questions.

\section{Statistical analysis}

A ranking order of motivational and decision-making factors was compiled, ranking from the factor with the highest percentage of 'important' or 'very important' to the lowest. Differences between CC and PP were calculated using a Fisher's exact test.

RPS scores were analysed as described by Meertens. ${ }^{20}$ Differences in mean scores were analysed using a linear regression model, adjusting for age, sex and healthcarerelated education or job. Frequencies were calculated for the multiple-choice questions on the experiences of PP and ethical issues. Differences in demographical characteristics were calculated using a $\chi^{2}$ test, differences between CHI models were calculated using a one-way analysis of variance for continuous parametric data and Kruskall-Wallis test for non-parametric data, and a $\chi^{2}$ test for categorical data. A $p$ value $\leq 0.05$ was considered statistically significant.

Calculations were made using SPSS V.23. ${ }^{21}$

\section{Patient and public involvement}

No patients were involved in this study. This study was designed to investigate healthy volunteers' opinions and preferences. Volunteers were not involved in the design or recruitment process. Interested participants were presented the results during a meeting, participants will be provided the research article after publication.

\section{RESULTS}

Survey was returned by 61 of $66 \mathrm{CHI}$ participants and 156 of 156 students. There were no missing answers in the questionnaires of PP, although many CC did return incomplete questionnaires. Nevertheless, since all questions were answered by at least $85 \%$ of controls, all questionnaires were included in the analysis (all survey outcomes are provided in online supplementary C).

Baseline characteristics and demographics for both PP and CC are in table 1 . The majority of PP $(67 \%)$ were students while participating in their trial. Most PP had not previously taken part in medical research $(72 \%)$ and $53 \%$ were employed or studying in a healthcare-related field. In both groups, the majority were female. CC were younger than PP $(\mathrm{p}<0.0001)$ and most were recruited from the medical faculty.
Table 1 Demographic characteristics of study participants

CHI

participants Controls

$(n=61)$

$(n=156)$

\begin{tabular}{lll}
\hline $\begin{array}{l}\text { Participation in trial for } \\
\text { Schistosomiasis }(\mathrm{n}=17)\end{array}$ & $16(26 \%)$ & N/A \\
Hookworm $(\mathrm{n}=26)$ & $22(36 \%)$ & \\
Malaria $(\mathrm{n}=23)$ & $23(38 \%)$ & \\
Sex & & \\
\hline Male & $24(39 \%)$ & $35(22 \%)$ \\
Female & $37(61 \%)$ & $35(22 \%)$ \\
\hline Missing & & $23(15 \%)$ \\
Age & & $3(2 \%)$ \\
$<18$ years & 0 & $145(93 \%)$ \\
18 to 24 years & $38(62 \%)$ & $8(5 \%)$ \\
25 to 30 years & $11(18 \%)$ & 0 \\
$>30$ years & $12(20 \%)$ & $156(100 \%)$ \\
\hline Employment & & \\
Student & $41(67 \%)$ \\
Working & $19(31 \%)$ \\
Other & $1(2 \%)$ & \\
\hline
\end{tabular}

Previously participated in

research

$\begin{array}{lll}\text { Yes } & 17(28 \%) & \text { N/A } \\ \text { No } & 44(72 \%) & \end{array}$

Employed in healthcare or healthcare-related study?

\begin{tabular}{lll} 
Yes & $32(53 \%)$ & $126(81 \%)$ \\
\hline No & $29(47 \%)$ & $30(19 \%)$ \\
$\begin{array}{l}\text { Would you participate in } \\
\text { one of these controlled } \\
\text { human infection trials? }\end{array}$ & & \\
Yes, both & N/A & $9(6 \%)$ \\
Yes, only malaria & & $35(22 \%)$ \\
Yes, only hookworm & & $4(3 \%)$ \\
No & & $108(69 \%)$ \\
\hline
\end{tabular}

$\mathrm{CHI}$, controlled human infection.

Of the CC, $69 \%$ would not participate in any of the CHI trials (referred to as $\mathrm{CN}$ ), $22 \%$ would only participate in the malaria trial, $3 \%$ only in the hookworm trial and $6 \%$ in both trials (CC willing to participate in a CHI trial referred to as $\mathrm{CP}$ ).

\section{Motivation}

Motivation was investigated both by ranking factors of importance and by identifying the single most important factor. PP considered 'contributing to science' as an important $(43 \%)$ or very important $(38 \%)$ motivating factor, followed by 'contributing to developing countries' (41\% important and $31 \%$ very important) and the 


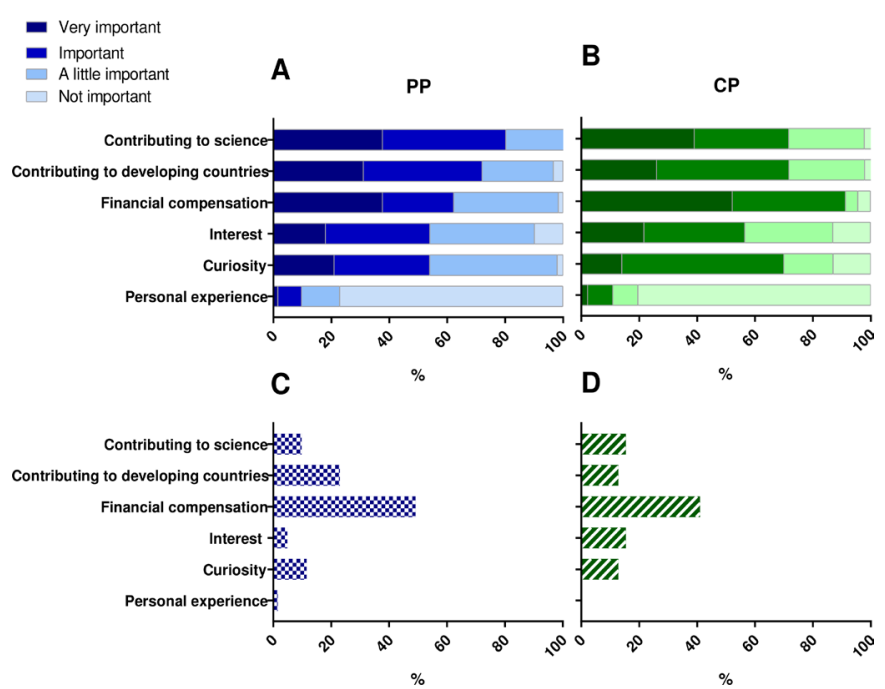

Figure 1 Ranking of motivational factors to participate in a $\mathrm{CHI}$ trial for PP (A) and $\mathrm{CP}(\mathrm{B})$. Single most important motivation factor for PP (C) and $\mathrm{CP}(\mathrm{D})$. CHI, controlled human infection; $\mathrm{CP}$, controls who would participate in a $\mathrm{CHI}$ trial; PP, actual $\mathrm{CHI}$ participants.

financial compensation (25\% important and $38 \%$ very important) (figure 1). However, when asked the single most important motivation, PP most often noted the financial compensation (49\%) followed by 'contributing to developing countries' (29\%) . There were no apparent differences in motivation for participants from different CHI models.

For CP the financial compensation was most often important (39\% important and 52\% very important, $\mathrm{p}=0.001$ for comparison between PP and CP), followed by 'contributing to science' (33\% important and 39\% very important, $\mathrm{p}=0.48$ ) and 'contributing to developing countries' (46\% important and $26 \%$ very important, $\mathrm{p}=0.9$ ). The single most important motivation was financial compensation for $41 \%$ of $\mathrm{CP}$ and 'contributing to science' and 'interest in the subject' for $15 \%$ each. The

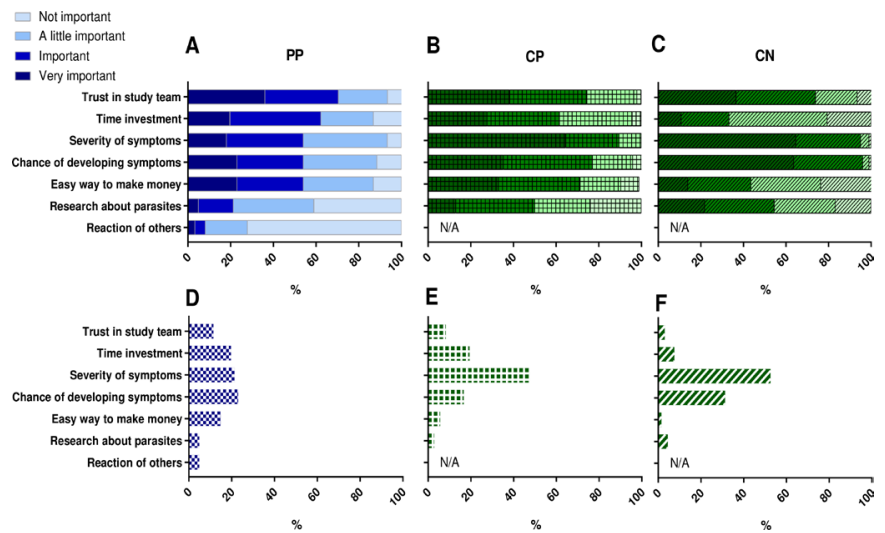

Figure 2 Ranking of factors considered in the decision to participate by PP (A), CP (B) and CN (C). The single most important factor in the decision to participate for PP (D), CP (E) and $\mathrm{CN}(\mathrm{F})$. CN, controls who would not participate in a $\mathrm{CHI}$ trial; $\mathrm{CP}$, controls who would participate in a $\mathrm{CHI}$ trial; $\mathrm{PP}$, actual controlled human infection trial participants. single most important factors were not distributed significantly different between PP and CP.

\section{Decision to participate}

PP most often found trust in the study team important in their decision to participate (34\% important and $36 \%$ very important) followed by the time investment $(43 \%$ important and $20 \%$ very important), severity of symptoms (36\% and 18\%), chance of developing symptoms $(31 \%$ and $23 \%$ ) and 'an easy way to make money' (31\% and 23\%). The single most important factor in the decision to participate was highly variable, including the chance of developing symptoms (23\%), severity of symptoms (21\%) and time investment (20\%).

CC most often considered the chance of developing symptoms and severity of symptoms important ( $<<0.001$ for comparison between PP and CC), with CP also considering the time investment and 'an easy way to make money'. The severity of symptoms was the single most important factor ( $47 \%$ for $\mathrm{CP}$ and $53 \%$ for $\mathrm{CN}$ ) (figure 2), which is significantly more often than for PP $(\mathrm{p}<0.001)$.

\section{Assessment of symptoms and risks}

The majority of PP (57 out of $61,93 \%$ ) considered the trial to be of no or little risk and the majority were not afraid of symptoms before the start of the trial (49 of 61, $80 \%$ ). For $10 \mathrm{PP}$, their fear of symptoms increased during the trial, mainly because they saw other volunteers with symptoms or as one volunteer stated, "we were working each other up the day of the malaria infection about the mosquito bites and what would happen". For the others, fear of symptoms declined $(n=8)$ or remained the same $(n=43)$. PP scored the symptoms they experienced during the trial on a scale of 0 to 10 , with 0 being no complaints at all, and 10 being complaints so severe they had to withdraw from the trial. The mean score was 2.85 (SD 2.7, range 0 to 10) for all models, with no significant differences between CHI models ( $\mathrm{p}=0.228$ ).

\section{Reaction of others}

Many (80\%) PP reported negative reactions about their trial participation, quoting reactions like: "Are you getting worms in your body?" or "You are taking a risk with your health". However, $64 \%$ also received positive reactions, such as "That's an important thing to support", "That is very interesting research to participate in" and "That's good money for little effort". The responses of third parties largely did not influence their decision to participate (93\%). All PP but one reported no outside pressure to participate in the study; the one exception was a participant who, while describing no pressure to initially participate, reported some during the study when the participant was unable to meet some of the logistical demands of the study. In response, the participant was offered the option of missing out on certain follow-up procedures in order to remain in the study for the primary endpoint, rather than dropping out 
A

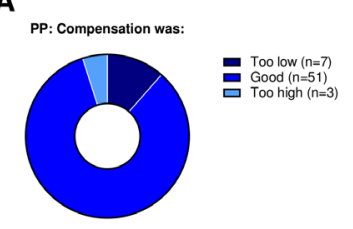

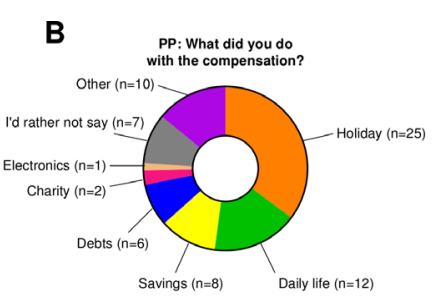

How do you view the compensation?

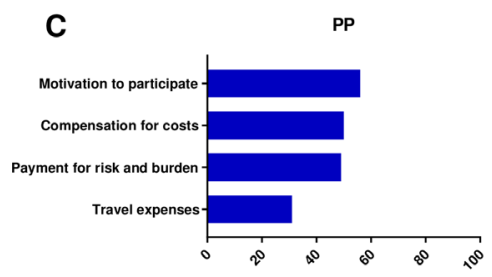

D cc
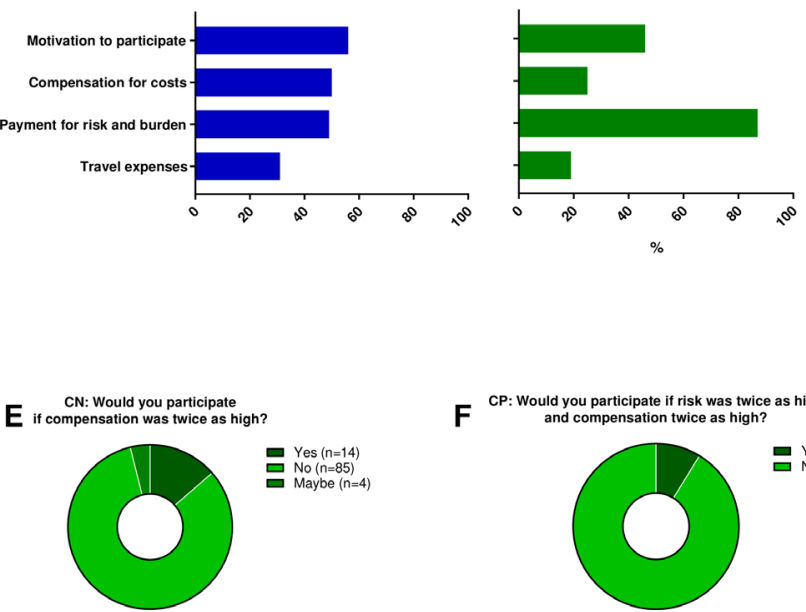

F

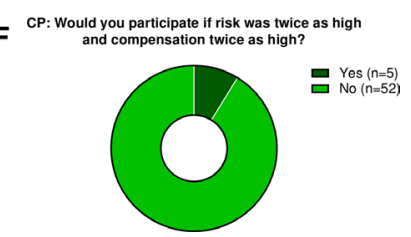

Figure 3 Opinion of PP ( $n=61)$ on the amount of financial compensation (A) and how they used the compensation (B). View of PP (C) and CC (D) on why financial compensation is offered (multiple answers could be given). Opinion of $\mathrm{CN}$ $(n=103)$ to change their mind if compensation was twice as high (E) and opinion of CP $(n=57)$ if the compensation was twice as high and risk was twice as high (F). CC,control group; CN, controls who would not participate in a $\mathrm{CHI}$ trial; $\mathrm{CP}$, controls who would participate in a $\mathrm{CHI}$ trial; PP, actual controlled human infection trial participants.

altogether. This participant described being glad to have been offered that proposition and was proud to have completed the study after all.

\section{Opinion on ethical issues}

$\mathrm{PP}$ and $\mathrm{CG}$ were asked their opinion about the concept of deliberate infection and the right to withdraw. For $77 \%$ of PP, it was considerably or very important to always be able to withdraw. However, $95 \%$ replied that they found it understandable that in a CHI trial immediate withdrawal is not always possible if this was done for their own safety or that it was acceptable if explained during the informed consent procedure. PP also found it acceptable for a physician to deliberately make them ill for the benefit of the trial $(100 \%)$. Some added that this was what they voluntarily signed up for, as long as possible symptoms were explained before the trial. CC generally had similar views: $94 \%$ felt it was understandable that it is not always possible to withdraw and $82 \%$ found it acceptable for a physician to deliberately make a person ill for the trial.

\section{Financial compensation}

Of the PP, 10 out of 61 would have participated without any financial compensation. The majority of PP (84\%) considered the compensation as good, and three considered it too high. PP most often spent the financial compensation on a holiday $(41 \%)$, followed by costs of daily life (20\%) and savings (18\%). PP view the compensation as an incentive to participate $(56 \%)$, compensation for costs (50\%) and payment for risk and burden (49\%). The majority of $\mathrm{CN}$ could not be convinced to participate for double the compensation $(86 \%)$ and only $3(3 \%)$ would change their mind about participation if both the compensation and the risks were doubled. CP were also unwilling to take more risk: only 5 of the $44(11 \%)$ would still participate if the risk was twice as high but compensation also twice as high (figure 3).

\section{Looking back at participation}

Remarkably, a large proportion (59\%) of PP felt they had gained benefits from their participation other than the financial compensation, like increased knowledge about the conduct of clinical trials or the disease for which they participated, the pride of having contributed to important research and the experience of going through a trial with the other participants and the study team. One volunteer stated that he had 'learnt to get up early in the morning and improve my daily rhythm'. Most $(84 \%)$ were proud of their participation, would advise others to participate $(89 \%)$ and would participate in a similar trial again (85\%) (figure $4 \mathrm{~A}$ ). In retrospect, $80 \%$ felt that the benefits of the study outweighed the burden they experienced, and of the $20 \%$ who did not, 3 out of 12 stated they had experienced so little discomfort that they did not have any burden. For $46 \%$ of volunteers, the symptoms met their expectations, $36 \%$ experienced fewer symptoms than expected and 20\% experienced more (figure 4B). Even those participants who had more symptoms than expected evaluated their participation positively: eight out of 12 felt proud of their participation and would advise others to participate, while 10 out of 12 would participate again (figure 4C).

\section{Risk propensity scale}

PP had a significantly higher risk propensity score than CC (estimated difference 0.9, $\mathrm{p}<0.001$ )) (figure 5). CP also scored significantly higher than CN (estimated difference $0.9, p=0.001$ ). No evidence for differences between participants from different CHI models, males or females or those with a healthcare-related job or education were observed.

\section{DISCUSSION}

This survey study is the first to quantitatively investigate the motivations and experiences of participants in CHI trials. These findings shed light onto the experiences and opinions of participants on issues that have been the subject of extensive ethical debate. 


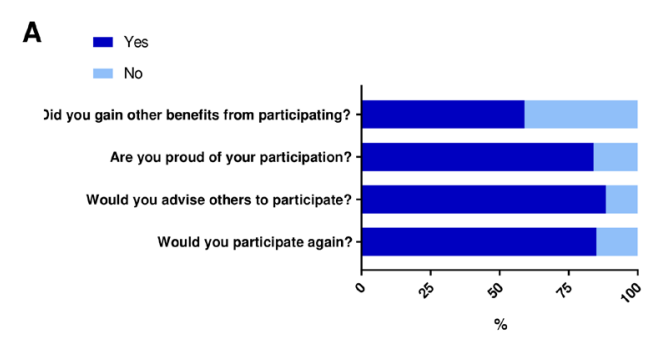

B

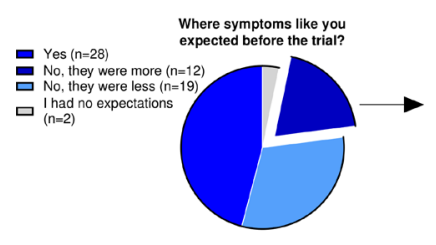

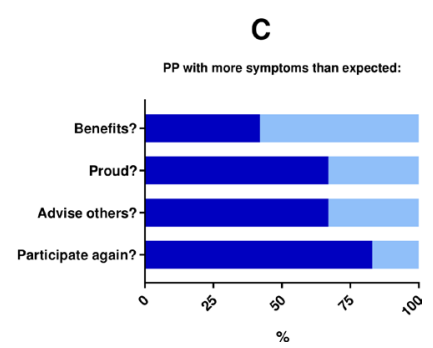

Figure 4 General evaluation of PP $(n=61)$ looking back at their participation $(A)$, assessment of symptoms when looking back (B) and general evaluation of PP who experienced more symptoms than expected (C). PP, controlled human infection trial participants.

We have found that, contrary to commonly mentioned fears, ${ }^{1022}$ the largest group of volunteers felt that contributing to science and to research benefitting developing countries was an important motivation. For $51 \%$ of PP the financial compensation was not the most important reason to take part. Interestingly, for $38 \%$ of PP financial compensation was not or only of little importance, and $10(16 \%)$ would have participated without any compensation. Our data convincingly shows that factors other than financial compensation are important motivators which are considered in the decision to participate.

A larger group of CG found the compensation important compared with PP, although as a single most important motivation for participation, proportions were similar. CG also gave more importance to the symptoms compared with PP. Possibly, the compensation is initially

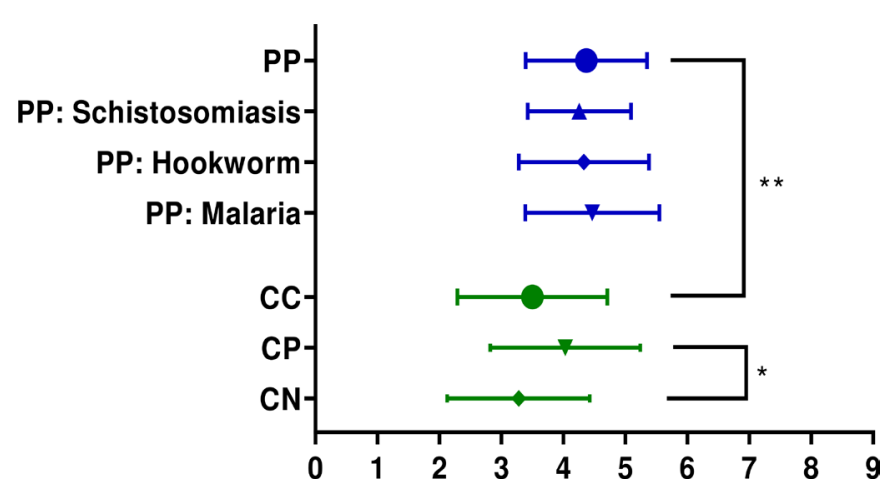

Figure 5 Risk Propensity Scale. Higher scores indicate a higher propensity to take risks. Symbols indicate mean, errors bars indicate SD. ${ }^{* *} p<0.001,{ }^{*} p=0.001$. CC, control group; $\mathrm{CN}$, controls who would not participate in a $\mathrm{CHI}$ trial; $\mathrm{CP}$, controls who would participate in a $\mathrm{CHI}$ trial; PP, actual controlled human infection trial participants. most important for a potential participant to be interested in the study, with motivations becoming more diverse after receiving more information about the study and through actual participation. In the decision-making process, CC gave more importance to the symptoms, which may reflect that during the first deliberations about participation, the symptoms are an important decider, whereas with more information, other factors are taken into account.

The motivations of CHI participants seem to be concurrent with findings in volunteers of phase I drug trials. Stunkel and Grady describe in a 2011 systematic review ${ }^{8}$ that although the financial compensation is usually necessary, it is not sufficient for participation, and note that risk is the deciding factor in participation. However, other large-scale studies in phase I drug-research participants, ${ }^{15}$ noted that money is the most important motivator in $60 \%$ of individuals, which is clearly more than we found. Possibly, the population (students, gender and age) might play a role in motivating factors as well as the nature of the trial. A survey of the motivations of individuals participating in Ebola and influenza vaccines is a good example of the latter, whereby almost $90 \%$ of participants found contributing to the health of others important. ${ }^{23}$ It is possible that both $\mathrm{CHI}$ trials, especially those researching vaccines for neglected tropical diseases and phase I trials for vaccines with similar expected public health benefits may attract volunteers with more altruistic motivations compared with phase I drug research in general.

Differences in population may also be reflected within CHI studies in different countries. Our Dutch PP were motivated by other factors than Kenyan participants of a CHMI trial, who were most often driven by the financial compensation and the healthcare provided by the trial staff. $^{18}$ The Kenyans were rewarded the wage of a day's work for each day of participation to make up for lost income. This was different for the Dutch PP, who have universal access to healthcare and receive compensation for time spent and travel expenses. Participants from both countries, however, showed little concern about trial risks and showed high levels of trust in the study team. In a qualitative study among US CHMI participants, ${ }^{17}$ the participants similarly describe little concerns about the risks, trust in the study team as important and mixed motivations for participation. The differences between the American, Kenyan and Dutch CHI participants illustrate the influence of cultural differences and healthcare organisation that remain important to address and separately investigate.

This study also provides more insight into the presence of undue influence by the financial compensation. We have found that a majority of PP used their compensation for leisure activities such as a vacation or put the money in their savings accounts. This indicates they do not have a direct financial need in daily life to take part but could spend the money for more luxury expenses. The CG also provides evidence that potential participants cannot be persuaded to participate for more money if 
they are not inclined to do so in the first place, or accept more risk for more money, even though the compensation is an important motivation to participate for them. We acknowledge that without any compensation, many PP would probably not participate but do conclude that the motivations of participants are varied and that the role of the financial compensation is not as important as presumed.

Another important issue in current debate is the acceptable risks and burden to participants and the risk-taking attitude of trial participants. This survey cannot answer what acceptable risks and burdens are, but can give important insight into what participants actually consider acceptable.

Both PP and CP scored higher on the RPS as compared with $\mathrm{CN}$. Interestingly, the scores in both groups were lower than those of the original validating study for the RPS which had a mean score of 4.63 (SD 1.23, range 2.00 to 7.00$),{ }^{20}$ suggesting that the RPS varies considerably between different populations. Possible symptoms and risks were an important reason for $\mathrm{CN}$ to decline participation, whereas CP and PP apparently weigh the symptoms but find them acceptable. This higher acceptance of possible risks matches the higher risk-taking propensity, but does not mean that risks and burden are not considered. Even the majority of participants who experienced more symptoms than expected look back positively on their participation, are proud of their participation and would participate again. Combined with the finding that the large majority of PP felt the benefits outweighed the burdens of the study, the majority would participate again and would advise others to do so too, and that many reported to have gained more benefits than the financial compensation alone, we conclude that at least for these studies, the balance of burdens and risks was acceptable to the volunteers.

This study did not specifically assess understanding and informed consent by the PP; however, some conclusions on the success of informed consent and voluntariness can be drawn. All participants but one reported no pressure to participate. Although a reporting bias cannot be excluded, $\mathrm{PP}$ were a heterogeneous group of volunteers with diverse backgrounds, none of which connected to the research department. Most participants also indicate that the symptoms experienced were as expected or less, showing they had adequate expectations before starting with the trial. This is confirmed by the fact that most PP reported no change or a decrease in their fear of developing symptoms during the study. We have found no suggestion of pressure to participate and generally conclude that PP were well informed about participation, although a more targeted survey would address this question more directly.

This survey also illustrates PP's and CC's views on other issues of ethical debate in CHI trials. The right to withdraw is considered very important by both groups; however, most, including $\mathrm{CN}$, agree that it is acceptable to put restrictions on this if done for the safety of the volunteer and agreed beforehand. The majority of CC did not express ethical concerns about the concept of deliberate infection as they believe that the research will be performed in a safe manner and that risks and benefits are adequately weighed, showing an apparent acceptance of this kind of research even by those who would not participate. This shows that if properly informed, participants are willing to accept some restrictions on the right to withdraw, highlighting the importance of complete and thorough informed consent procedures.

Recall bias may have distorted some of the answers to the questionnaires because of the long lag time between completion of the CHI trial and filling out the survey for some volunteers. Some answers to questions in the PP group may also have been influenced by participation in the trial. In addition, social desirability and missing answers may have confounded the results, although surveys were processed anonymously and missing answers were evenly distributed among the questions. Notwithstanding, this study has included a reasonably large number of CHI participants compared with previous studies and covers several different CHI models, thereby improving generalisability.

The use of the control group has several limitations. The control group of students may not be a complete representation of the participant population as it is more homogeneous in age, education and healthcare background than the actual participants which impairs generalisability. Controls were also offered a hypothetical participation, which may not be comparable to the actual decision to take part. However, participants are largely selected from the same population and this control group represents two-thirds of trial participants. We thus believe that the comparison is still of value.

\section{CONCLUSION}

As the first study to quantitatively investigate the motivations and perceptions of participants, this survey is a crucial addition to the ongoing debate on CHI trials. This study is among the first to add the voice of participants to the current debate. We found that the motivation of CHI participants is highly varied, with significant importance for altruistic motivations. Participants are able to make a balanced appraisal of risks and burdens that results in a mostly satisfactory experience of participation for them. Based on these findings, we propose that the current image of the CHI participant as 'money-oriented risktaker' is not accurate and may have to be nuanced to the CHI participant as 'deliberate decision-maker'.

Acknowledgements The authors are grateful to Jacqueline Janse, MSc, for her help in developing the questionnaire and Castor database, and to Clarize de Korne, MSc, for her fruitful discussions and ideas on this subject. M-AH was supported by Dioraphte Foundation for another trial. Dioraphte Foundation was not involved in the design, execution or publication of the present study.

Contributors $\mathrm{M}$-AH devised the study and surveys, collected the data, analysed the data and drafted the manuscript. MdV critically reviewed the surveys, analysed the data and critically reviewed the manuscript. MR supervised the clinical trials, critically reviewed the surveys, analysed the data and critically reviewed the 
manuscript. JJ aided in developing the questionnaires and built the Castor database used for data collection and analysis. CK commented on data collected in informal discussions and gave input for ideas in the manuscript.

Funding The authors have not declared a specific grant for this research from any funding agency in the public, commercial or not-for-profit sectors.

Competing interests None declared.

Patient and public involvement Patients and/or the public were not involved in the design, or conduct, or reporting, or dissemination plans of this research.

Patient consent for publication Not required.

Ethics approval The institutional review board of the Leiden University Medical Center where the study was performed reviewed the protocol and provided ethical approval (P18.203).

Provenance and peer review Not commissioned; externally peer reviewed.

Data availability statement All data relevant to the study are included in the article or uploaded as supplementary information.

Open access This is an open access article distributed in accordance with the Creative Commons Attribution Non Commercial (CC BY-NC 4.0) license, which permits others to distribute, remix, adapt, build upon this work non-commercially, and license their derivative works on different terms, provided the original work is properly cited, appropriate credit is given, any changes made indicated, and the use is non-commercial. See: http://creativecommons.org/licenses/by-nc/4.0/.

\section{ORCID iD}

Marie-Astrid Hoogerwerf http://orcid.org/0000-0002-3088-3380

\section{REFERENCES}

1 Roestenberg M, Hoogerwerf M-A, Ferreira DM, et al. Experimental infection of human volunteers. Lancet Infect Dis 2018;18:e312-22.

2 Darton TC, Blohmke CJ, Moorthy VS, et al. Design, recruitment, and microbiological considerations in human challenge studies. Lancet Infect Dis 2015;15:840-51.

3 Stanisic DI, McCarthy JS, Good MF. Controlled human malaria infection: applications, advances, and challenges. Infect Immun 2018;86. doi:10.1128/IAI.00479-17. [Epub ahead of print: 19 Dec 2017].

4 Chen WH, Cohen MB, Kirkpatrick BD, et al. Single-Dose live oral cholera vaccine CVD 103-HgR protects against human experimental infection with Vibrio cholerae O1 El Tor. Clin Infect Dis 2016;62:1329-35.

5 Bambery B, Selgelid M, Weijer C, et al. Ethical criteria for human challenge studies in infectious diseases. Public Health Ethics 2016;9:92-103.
6 Hope T, McMillan J. Challenge studies of human volunteers: ethical issues. J Med Ethics 2004;30:110-6.

7 Miller FG, Grady C. The ethical challenge of infection-inducing challenge experiments. Clin Infect Dis 2001;33:1028-33.

8 Stunkel L, Grady C. More than the money: a review of the literature examining healthy volunteer motivations. Contemp Clin Trials 2011:32:342-52.

9 Srinivasan S, Johari V. Consultations on human infection studies in India: do people's voices really count? Indian J Med Ethics 2019;4:234-7.

10 Elliott C, Abadie R. Exploiting a research underclass in phase 1 clinical trials. N Engl J Med 2008;358:2316-7.

11 Evers DL, Fowler CB, Mason JT, et al. Deliberate microbial infection research reveals limitations to current safety protections of healthy human subjects. Sci Eng Ethics 2015;21:1049-64.

12 Pieters MS, Jennekens-Schinkel A, Schoemaker HC, et al. SelfSelection for personality variables among healthy volunteers. $\mathrm{Br} J$ Clin Pharmacol 1992;33:101-6.

13 Farré M, Lamas X, Camí J. Sensation seeking amongst healthy volunteers participating in phase I clinical trials. Br J Clin Pharmacol 1995;39:405-9.

14 Chen SC, Sinaii N, Bedarida G, et al. Phase 1 healthy volunteer willingness to participate and enrollment preferences. Clin Trials 2017;14:537-46.

15 Grady C, Bedarida G, Sinaii N, et al. Motivations, enrollment decisions, and socio-demographic characteristics of healthy volunteers in phase 1 research. Clin Trials 2017;14:526-36.

16 Langenberg MCC, Hoogerwerf M-A, Janse JJ, et al. Katayama syndrome without Schistosoma mansoni eggs. Ann Intern Med 2019;170:732.

17 Kraft SA, Duenas DM, Kublin JG, et al. Exploring ethical concerns about human challenge studies: a qualitative study of controlled human malaria infection study participants motivations and attitudes. J Empir Res Hum Res Ethics 2019;14:1556264618820219:49-60.

18 Njue M, Njuguna P, Kapulu MC, et al. Ethical considerations in controlled human malaria infection studies in low resource settings: experiences and perceptions of study participants in a malaria challenge study in Kenya. Wellcome Open Res 2018;3:39.

19 Castor Electronic Data Capture [program]. Amsterdam 2016.

20 Meertens RM, Lion R. Measuring an individual's tendency to take risks: the risk propensity scale. J App/ Soc Psychol 2008;38:1506-20.

21 IBM Corp. IBM SPSS Statistics for Windows, Version 23.0 [program. Armonk NY: IBM Corp, 2015.

22 Stones M, McMillan J. Payment for participation in research: a pursuit for the poor? J Med Ethics 2010;36:34-6.

23 Cattapan A, Browne K, Halperin DM, et al. Motivation for participating in phase 1 vaccine trials: comparison of an influenza and an Ebola randomized controlled trial. Vaccine 2019;37:289-95. 